\title{
From Business Intelligence Insights to Actions: A Methodology for Closing the Sense-and-Respond Loop in the Adaptive Enterprise
}

\author{
Soroosh Nalchigar and Eric Yu \\ Department of Computer Science, University of Toronto \\ \{soroosh, eric\}@cs.toronto.edu
}

\begin{abstract}
Business Intelligence (BI) and analytics play a critical role in modern businesses by assisting them to gain insights about internal operations and the external environment and to make timely data-driven decisions. Actions resulting from these insights often require changes to various parts of the enterprise. A significant challenge in these contexts is to systematically connect and coordinate the BI-driven insights with consequent enterprise decisions and actions. This paper proposes a methodology for closing the gap between what an enterprise senses from BI-driven insights and its response actions and changes. This methodology adopts and synthesizes existing modeling frameworks, mainly $i^{*}$ and the Business Intelligence Model (BIM), to provide a coherent step-bystep way of connecting the sensed signals of the enterprise to subsequent responses, and hence to make BI and analytics more actionable and understandable. Applicability of the proposed methodology is illustrated in a case scenario.
\end{abstract}

Keywords: Business Intelligence, Data Analytics, Adaptive Enterprise, Sense-and-Respond, Modeling framework.

\section{Introduction}

Business Intelligence (BI) and analytics have gained a great deal of attention from both academic and business communities over past two decades [5]. These systems serve as sensing mechanisms of the enterprises by providing insights about their strategic goals, operations, performance, as well as the external environment, and assist them to respond to critical situations and take advantage of emerging opportunities. However, a critical challenge to the adoption of these systems is the lack of understanding of how to use analytics and the insights resulting from them to improve the business [20, 31]. In other words, a leading challenge in using insights from BI is to connect them to business operations and hence shorten the latency. Latency is defined as the time taken from something happening within or around the enterprise to the moment when it reacts to it [26].

J. Grabis et al. (Eds.): PoEM 2013, LNBIP 165, pp. 114-128, 2013.

(C) IFIP International Federation for Information Processing 2013 
BI-driven insights can stimulate corrective actions and changes within various layers and different parts of an enterprise 31]. For example, a manufacturing company that uses BI solutions may find an increasing trend in the production costs. To reduce the costs, this company may change the manufacturing layout or may decide to modify the inventory ordering policies. A hospital which uses analytics may see a long waiting time in the reception room and decides to increase the number of physicians and/or change their work schedules. A bank whose web analytics show a decreasing trend in number of online banking users and transactions, may decide to change the interface and the web-page design to make it more user-friendly and increase its ease of use. In all of these contexts, in order to trigger new changes across the enterprise and to take action at the right time, BI-driven insights must be easy to interpret, linked to business strategy, and embedded into organizational processes [20].

To tackle this problem, we propose a model-based methodology for closing the gap between what an enterprises senses from the BI insights and their response actions and changes. The proposed methodology adopts and synthesize several requirements engineering modeling frameworks, including $i^{*}$ [28, 29, 32] and Business Intelligence Model (BIM) [8, 3, 1, 14], to provide a systematic and coherent way of connecting the BI-driven insights to the consequent business action and changes. Our methodology assists business users to conceptualize strategic goals and to design the sensing mechanisms for monitoring those goals. It helps them generate and analyze the alternative response actions, to make trade-offs between them, and to find the most suitable next action. Finally, the methodology aids analysts to obtain new requirements to adapt the BI system to the new enterprise context resulting from the sense-and-respond loop.

The rest of this paper is structured as follows. Section 2 reviews related work and highlights the need for the proposed methodology. Section 3 provides an example to illustrate and motivate the research problem. Section 4 presents the proposed methodology and illustrates its applicability on the motivating example. The paper ends with concluding remarks and future work directions in Section 5 .

\section{Related Work}

In this section we review related works and published papers and clarify the position of this paper within previous works.

Managing change and facilitating adaptiveness has been a continuous theme in enterprise architecture [31]. In 1995, Stephan Haeckel proposed a sense-andrespond model of adaptive enterprise as a new management tool for achieving competitive advantage in dynamic business environment [13]. An adaptive enterprise has the ability to sense environmental signals sufficiently and to translate them quickly into meaning and subsequent actions [11]. Haeckel described the transformation from make-and-sell enterprise, characterized by high-volume/low cost mass production, to sense-and-respond enterprise which is modular, fluid, and effectively responds to dynamic and non-linear changes. He emphasized 
to design adaptive enterprise based on sense, interpret, decide, and act loops 13, 11, 12. Inspired by Haeckel's works, Buckley et al. [4] and Kapoor et al. 18] proposed a technical framework for sense and respond business and performance management. Their framework utilizes and integrates optimization and analytics models to enable proactive management and control of business resources. They implemented prototypes and showed applicability of the proposed framework for adaptive inventory management and demand/supply conditioning in two pilot projects in IBM divisions. This line of research was continued by Chowdhary et al. 6], where they applied model-driven techniques to IBM Business Performance Management (BPM) solution and illustrated their framework in a scenario of a pilot project. Also, Kapoor et al. [19] proposed a model-driven development framework for a sense-and-respond supply chain.

Nguyen et al. 23] proposed a real time service-oriented BI architecture (called SARESA) that covers sense and respond loop and thereby aims to decrease the reaction time. They implemented and illustrated their approach in a mobile phone fraud detection application which analyzes the phone calls and issues relevant alarms in fraud cases. Panian [26] discussed the notion of real-time decisioning as an analytic approach that allows enterprise to automate the "next best action" based on performance goals. He proposed six general steps which needs to be accomplished in order to make real-time decisioning work. In another work, Panian 25] discussed the characteristics and benefits of Service-oriented Architecture (SOA) and suggested to use BI solutions as Web services in an SOA environment.

Although these works contribute to the field and aim to increase enterprise adaptiveness, the proposed solutions are either in the form of technical architectures for specific settings or in terms of a set of general managerial principles and guidelines. Indeed, there is a lack of enterprise models that allow for analysis of adaptiveness as well as a systematic step-by-step way of closing the sense-andrespond loop in business contexts. Yu et al. 31] discussed various research challenges and directions about adaptive enterprise architecture. They mention the lack of a framework that includes collection of modeling constructs from various frameworks (e.g., goal modeling [7], social modeling [32], system dynamics, and BIM framework) in order to model and analyze the change and adaptiveness of the enterprise.

Recent efforts have attempted to introduce business-level modeling to provide a higher level abstraction for BI. The BIM framework [3] conceptualizes the business operations, strategies, situations and performance indicators in a way that can assist exploiting the huge amounts of data collected by enterprise. An empirical evaluation of the BIM in a Toronto hospital reported that BIM models enhance communication between technical and business stakeholders and support implementation in BI projects [2]. Horkoff et al. [14] proposed a detailed and precise definition of BIM concepts and a methodology for development of these models. It reviewed and extended BIM reasoning techniques with composite and incomplete indicators, summarized information requirements and connected them to the proposed methodology. Horkoff et al. [15] used OWL Description 
Logic (DL) to provide a formal semantics for BIM language. The semantics serves as a connection to DL reasoners in order to assist various "what-if?" analyzes, to detect inconsistencies, and to automatically classify defined concepts relative to existing concepts.

While the BIM modeling framework assists enterprises to utilize their data and provides them a better understanding of business, its connection to the action side has not been elaborated. In other words, BIM framework can help business users to sense and partially interpret how well they are doing with regarding to strategic goals, but it does not address the decide-and-act side of the sense-andrespond loop. We believe that business analysts need a more complete modeling framework which can assist them to achieve enterprise adaptiveness by closing the sense-and-respond loop thus making BI and analytics more actionable. The main contribution of this paper is to fill this gap by adopting and synthesizing the BIM framework with other frameworks in order to bridge the gap between BI-driven insights and consequent enterprise actions.

\section{Motivating Example}

In this section we consider a hypothetical example of a North American company, TVStars Inc., in the market of electronics, whose main products are TV and Home Theatre. The company has the vision of becoming a prestigious market leader recognized for its high performance products. The CEO and other senior managers of the company understand that the current business market in which they are operating is highly competitive, dynamic, and rapidly changing. To survive and thrive in this environment, they believe the company must be able to sense and adapt to changing market needs, to predict and cope with emerging threats, and still be able to monitor and satisfy stakeholder needs.

To monitor business performance and to enable managers to make data-driven and fact-based decisions, the company has recently deployed a BI solution. In this system, the performance of the business is made visible to responsible managerial positions and analysts by using monitoring dashboards and graphical display functions in the user interface. Using the BI system, the company has found a downward trend in the number of new and loyal customers. Moreover, the analytics show that the manufacturing costs of the company are above the planned targets. Having these insights, the managers and other BI users aim to improve the business performance. In this condition, they have faced a critical challenge which is lack of understanding how to use the output of BI system to improve business performance. They find it challenging to derive suitable corrective actions from the insights provided by the BI system. They want to know: What are the possible business actions (enterprise responses) to deal with the current bad performance in the indicators? Is it a good idea to target a new market in Europe and to sell our products there? Should the company change the product packaging policies from outsourcing to insourcing and hence reduce the manufacturing costs? Which of these is the most suitable business actions in this situation? After following/implementing it, how can we monitor the improvements? What are the new requirements for the current 


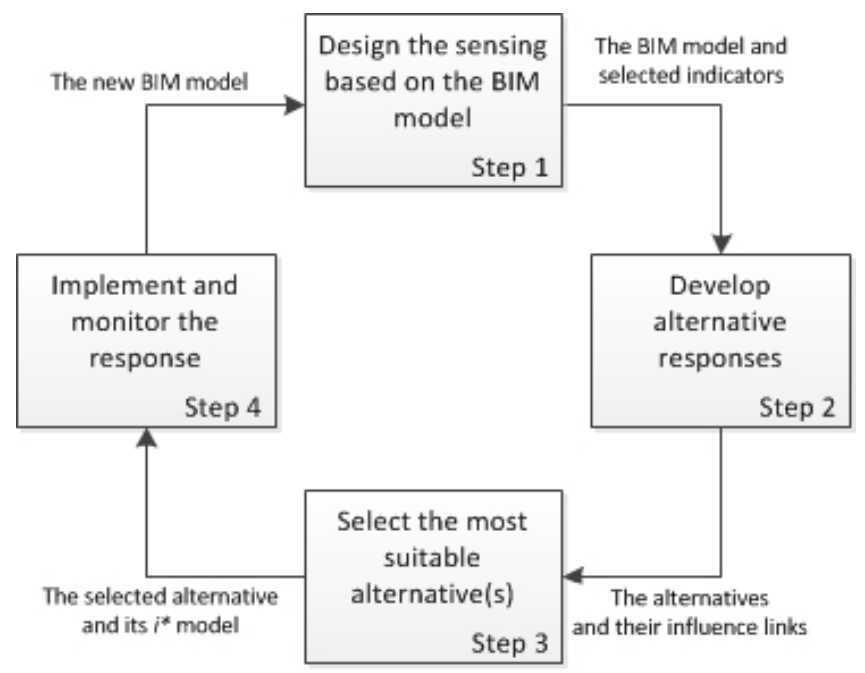

Fig. 1. Overview of the proposed methodology

BI system? How changes and enhancements to the BI system should be included as part of the response action? The main contribution of this paper is to propose a modeling framework that assist such organizations to facilitate coping with these challenges.

\section{Proposed Method}

In this section, we present the proposed methodology and show its application on the hypothetical example described in Section 3. Figure 1 shows an overview of the methodology and the inputs and outputs of the steps.

Step 1: Design the sensing based on the BIM model. In this phase, the first activity is to construct a business schema to support modeling and analysis of the enterprise and its performance. For this purpose, we use the BIM framework because it assists business users to utilize and make sense of huge amounts of data about the enterprise and its external environment. This framework provides business analysts with a modeling and query language to reason about business objectives, strategies, situations, tasks and performance indicators and hence to realize how well they are doing with respect to strategic goals and objectives. BIM draws upon well-known business concepts which makes it business-friendly and understandable for users. A methodology for constructing BIM models has been proposed in [14].

A BIM schema provides a picture of the goals structure of the enterprise along with the corresponding indicators and shows how well they are met. The BIM modeling concepts are adopted and synthesized from business and management literature and also requirements engineering sources, e.g. Balanced Scorecard and 


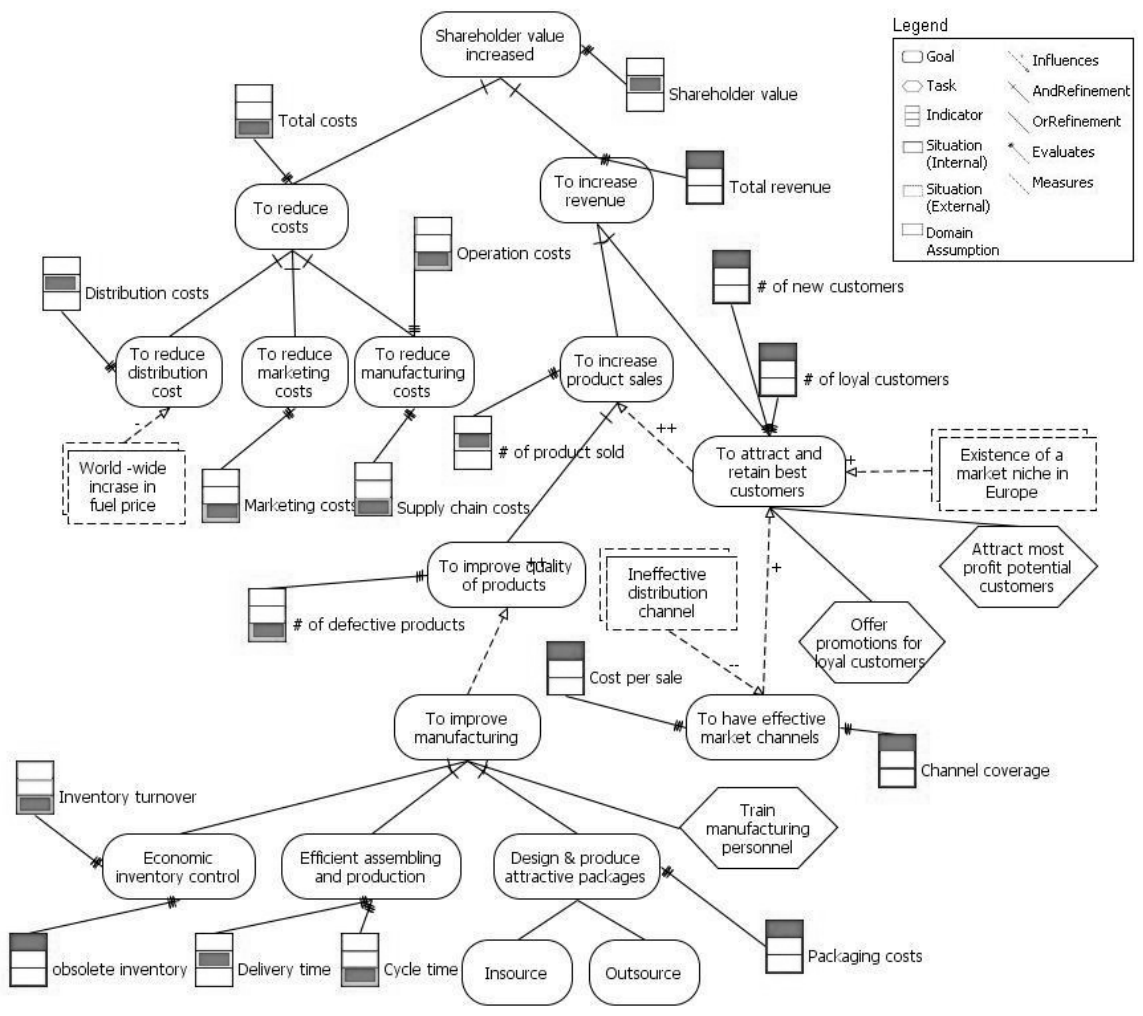

Fig. 2. Part of the TVStars BIM schema

Strategy Maps 17, 16], SWOT analysis (strengths, weaknesses, opportunities, and threats) 10], and goal-oriented requirements engineering 29, 9]. Reference 8] presents a full list of the concepts, definitions and examples of BIM.

Figure 2 shows part of the BIM schema of TVStars Inc. company, where the goal, influences and relationships, indicators (red on top, yellow on middle, green on bottom), and situations are modelled. The top level goal of the company is shareholder value increased. To achieve this goal, the company looks to meet the goals to reduce costs and to increase revenue. Moreover, to control the performance, the company have defined indicator(s) for each goal, e.g., number of loyal customers and number of new customers for the goal to attract and retain best customers. It shows how the organizational goals are refined into alternative actions that are means toward achieving those goals, e.g., a possible way to attract and retain best customers is to offer promotions for loyal customers. In addition, this model shows the external situations that are affecting the company's goals, e.g., ineffective distribution channel of the company, negatively affects the goal to increase product sales. 
After constructing the BIM model, the next activity in the proposed methodology is to analyze the indicators and select those for which the current performance is not excellent (red and yellow zones). This activity results in a set of indicators for which improvements and corrective actions (i.e., enterprise response) are needed. Analyzing the indicators in the BIM model is part of the sensing step of adaptive enterprise loop and assists business people to understand how well they are doing with regarding to their strategic goals and what/where are the possible areas of improvements.

At the current time, using the BIM model in Figure2, the TVStars Inc. senses that it has an average performance (yellow zone) in meeting its top goal. Moreover, the company realizes that the current performance with regarding to the goals to increase revenue and to attract and retain best customers are deficient, since the corresponding indicators are red. Also, the business analysts find that the total costs indicator is below the threshold and hence the corresponding indicator is in green zone, i.e., satisfactory level of costs. Having this model, and considering the competitive dynamic business market, the TVStars Inc. decides to undergo changes and take new course of actions in order to adapt and improve performance in the red/yellow indicators, e.g., number of product sold, number of new customers, and total revenue.

The main output of this phase, is an AS-IS BIM model of the enterprise along with a set of selected indicators for which the users want to improve the performance.

Step 2: Develop alternative responses. In this step, a set of alternative business actions are developed to deal with the poor performance in the indicators from previous step. These actions are potential future responses of the enterprise to what it has sensed. They could be in various business layers, involve different actors, and require different time, resources, and skills to be completed. The analyst adds these alternatives as new goals to the AS-IS BIM model from previous step.

After adding new alternatives, the next activity is to examine how each alternative is going to influence the other strategic goals of the enterprise. In particular, in order to model and consider the side-effects of the each alternative response, the analyst should examine how each alternative will impact the goals for which the current performance is excellent (green zone indicators). New influence links should be added to the BIM model to represents the possible effects of each alternative to other goals. These links facilitate the trade-off and decision making process among alternatives. At this step, the analysts can use the existing reasoning techniques on the BIM models to examine and answer a variety of strategic and analysis questions about effects of alternatives on the top goals of the enterprise. They can perform reasoning on the hierarchy of goals in order to evaluate different strategies for the satisfaction of top goals (forward reasoning), to evaluate the optimal input values leading to achievement of desired top goals (backward reasoning), as well as to perform analysis on goals inconsistencies and conflicts. Methods and techniques for reasoning with BIM models have been presented in $[1,14,3,2]$. 


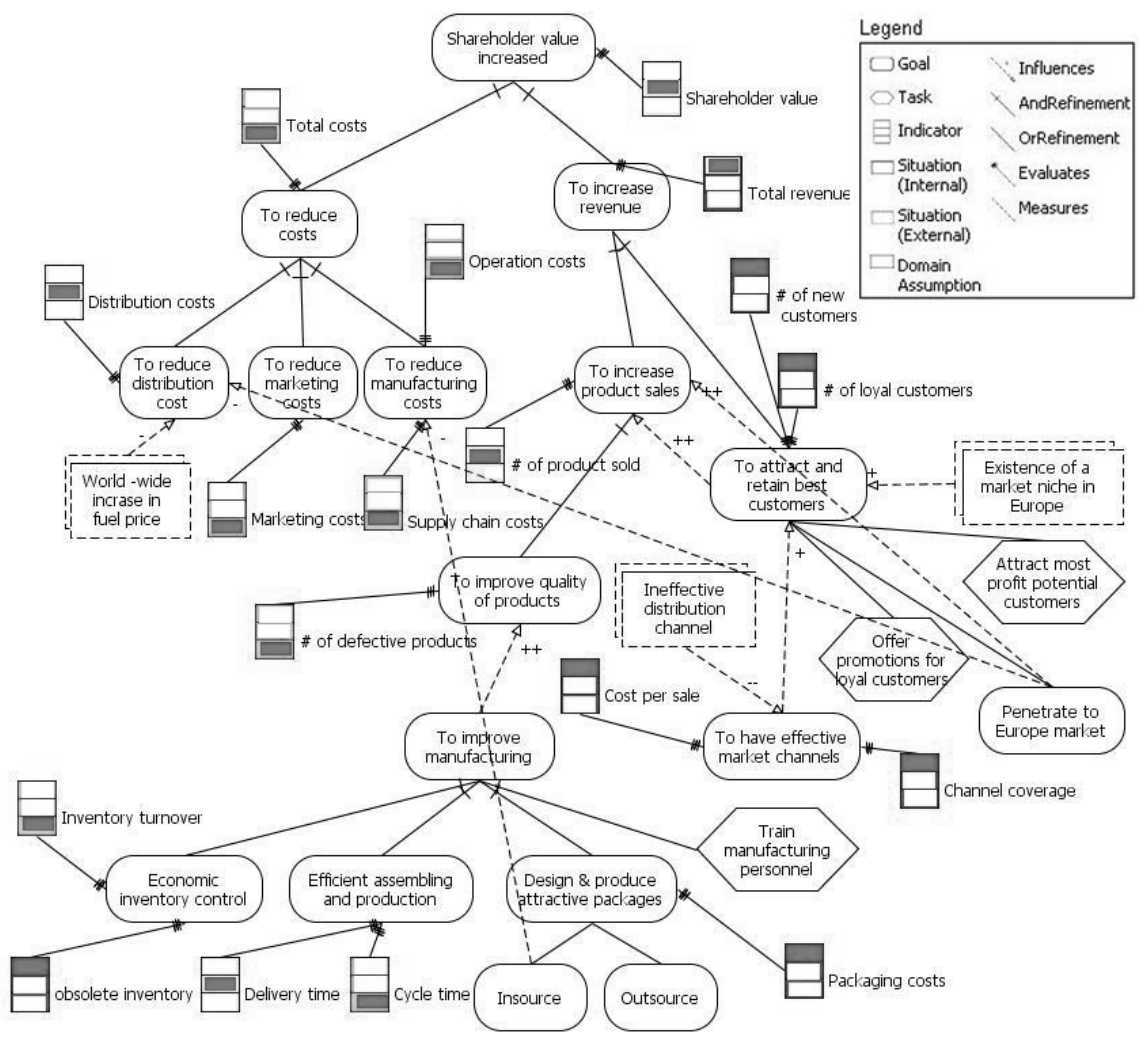

Fig. 3. BIM schema including the alternatives and their influence links

Figure 3 shows part of the new BIM schema for TVStars Inc. company. To generate this model, the BI analysts and the board of directors of the company examine and investigate the red and yellow zone indicators from previous step and after few meetings and discussions, they generate two alternative solutions that they believe could improve the indicators. Their first option is to penetrate to a new market in Europe. They believe that in this way they can increase number of new and loyal customers and hence increase the total revenue. Their second option is to change from outsourcing the package production to insource strategy, in which the company will design and produce the packages on its own. The analysts believe by insourcing the packaging of products, they will reduce the total costs of the company. Figure 3 also includes the new influence links of the alternative, showing that how these alternative will affect other strategic goals, e.g. penetrating to Europe market negatively affects the goal to reduce distribution costs.

The main output of this step is a BIM model which includes the alternatives along with their influence links to strategic goals. 


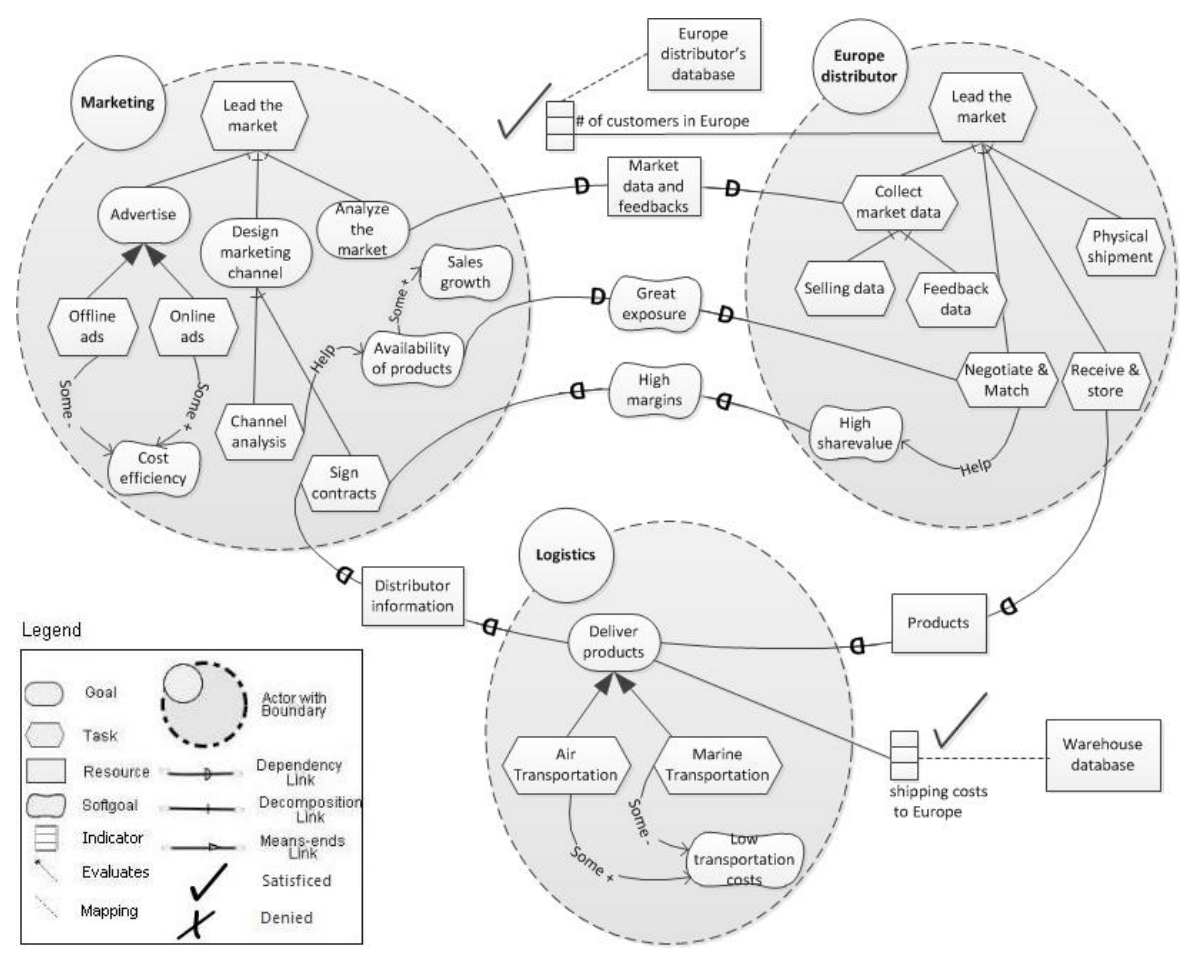

Fig. 4. $i^{*}$ model representing the first alternative

Step 3: Select the most suitable alternative(s). In this step, the decision among alternative responses of the enterprise is made and the best action(s) is chosen to be implemented. To facilitate the organizational decision making process, $i^{*}$ modeling framework is used to analyze each of the alternative responses. $i^{*}$ models assist the analyst to provide a detailed picture of how the corresponding organizational setting would look like when the given alternative is chosen and implemented. In other words, these models are snapshots of the enterprise once the response action is adopted and help the enterprise users to know how the alternative will change the settings, e.g., new actors in the system, new dependencies and the potential TO-BE socio-technical context.

$i^{*}$ models show the goal structure of social actors involved in the system and depict how the actors depend on each other to achieve their goals. Reference [30] reviews the recent applications of this modeling framework in practical industrial and business settings. Figure 4 shows the $i^{*}$ model constructed for the first response alternative for TVStars. Using this model, the decision makers realize that if they decide to sell the products in Europe, a new actor Europe distributor would be part of the system. The Marketing department depends on this distributor to have a great exposure in the market which helps to achieve the softgoal availability of products and hence sale growth. The model shows them that the distributor depends on the marketing department to provide high margins, so he 
can achieve his internal softgoal high sharevalues. Also, the marketing department has a resource dependency to the Europe distributor to provide market data and feedbacks from European customers. Moreover, the analysts find that the Europe distributor will depend on the logistics department of the company to provide the products. In addition to external dependencies, the $i^{*}$ model shows them internal goals of each actor and indicates decompositions and various ways of achieving those goals.

Figure [5] shows the $i^{*}$ model constructed for the second alternative. It indicates that if the company follows the second alternative, the insource packaging task would be done as an internal task within the authority of Manufacturing manager. Also, it shows different productions strategies and their influences on the softgoals of manufacturing department.

After developing these models, the next activity of the proposed methodology is to decide about the new indicators that the enterprise needs to measure after implementing each alternative. This will assist enterprises to monitor, after implementation of an alternative, how well it is working and allows them to adapt again if the performance is not satisfactory. These indicators are added to the $i^{*}$ models since these models are already showing the details of each alternative and can facilitate deciding about new indicators. The "measures" and "evaluates" links from the BIM meta model are used to connect the new indicators to the goals, tasks or the dependency links in the $i^{*}$ model. Now, by having the new indicators in the $i^{*}$ model, the analysts can examine if an indicator is accessible/computable or not. To facilitate that, the analyst connects each of the new indicators to the related existing data sources (as resources in the $i^{*}$ model). The connection between indicators and data sources elements are made using mapping elements from the Conceptual Integration Modeling (CIM) framework 27]. Another way of making this connection is to use the traceability links (e.g., satisfiability) proposed in [22, 21] to connect each new indicator to the data warehouse schema of the enterprise. The accessibility/computability of indicators are used as a decision criteria while trying to find the most suitable alternative. If an analyst finds that there is not available data for measuring an indicator, this could result in removing the corresponding alternative from possible choices.

The last activity in this step is to select the most suitable alternative. As a set of criteria for this decision making, analysts and business actors consider the influence links that were developed in the second step of the methodology, the $i^{*}$ models, and finally the feasibility of measuring the corresponding indicator $(\mathrm{s})$ for each alternative.

Following the scenario of TVStars Inc. company, the analysts add two new indicators to the model in Figure 4 number of customers in Europe and shipping costs to Europe believing that these indicators are necessary for measuring the performance of this alternative. The analyst finds that the first alternative could be calculated from the European distributor's database and the second one could be computed from the company's warehouse database which is already operating in the logistics department. Hence, he marks these indicators as satisfied in the 


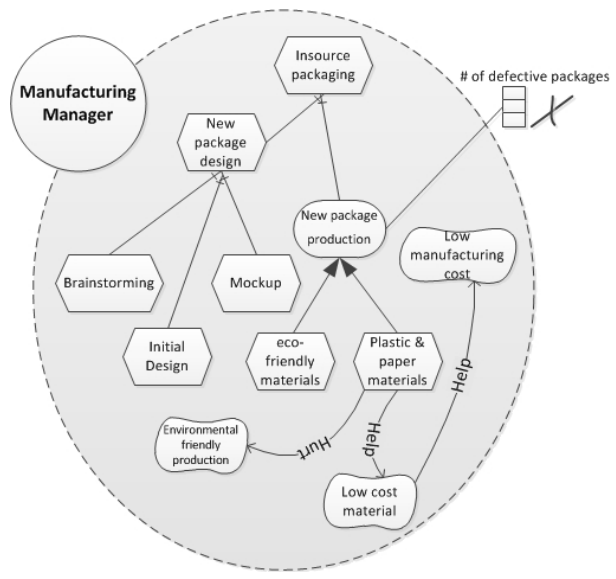

Fig. 5. $i^{*}$ model representing the second alternative (See Figure 4 for legend)

model. Regarding the second alternative, the analysts realize that at the current stage, the databases of the company does not provide the required data for computing the indicator number of defective packages. Hence, he marks this indicator as denied (See Figure 5). Having this analysis and also by considering the estimated influences of the alternatives on the other strategic goals (See Figure 3), the analysts conclude that first alternative is more suitable for the current situation of TVStars Inc. company. Hence, they decide to follow the first alternative as the next most suitable action (i.e., the response to what they had sensed).

The main output of this step is the chosen alternative, its corresponding $i^{*}$ model including new indicators, and connections to the data warehouse schema.

Step 4: Implement and monitor the response. The last step of the methodology includes the implementation of the chosen alternative, which is the enterprise's response to what it had sensed in the first step. The $i^{*}$ models developed in previous step could be used to assist in the implementation. Besides, the enterprise can use the change management guidelines and principles existing in the literature. This step of the methodology also includes modifying the BI system to the new settings (i.e., chosen alternative) so the enterprise keeps track of how well it is doing with regard to its response(s). The analysts construct the TO-BE BIM model which includes the selected alternative from previous step and its associated indicators. This BIM model includes the new requirements for the BI system of the enterprise.

In the last step, the TVStars Inc. company penetrates to the Europe market and starts selling the products there. The company also modifies the BI system to include the new requirements, i.e., measuring the indicators related to the first alternative. After implementing the alternative and updating the BI system, the TVStars Inc. company has completed an iteration of the sense-and-respond loop. 
To summarize, this methodology adopts and synthesizes a set of modeling frameworks to provide a systematic way of closing the sense-and-response loops of an adaptive enterprise. The methodology includes conceptualizing and modeling the strategic goals and objectives of the enterprise and thereby sensing how well the current performance is w.r.t. each of them. This is covered in the first step of the methodology (see Figure 2). The proposed methodology models various response alternatives to a given output of BIM model, as well as their associated indicators (see Figure 3). Our methodology includes modeling the social interaction and dependencies between various actors in an adaptive enterprise context. It aids business users to make trade-off between these alternatives, and finally to decide about the most suitable one (see Figures 4 and 5 ). Besides, it serves as a way to model the new requirements and adapt the BI system in a changing dynamic environment (see the last step of the methodology). By connecting the sensed signals of the enterprise to subsequent responses, we believe the proposed methodology can make BI and analytics more actionable and understandable for business users.

\section{Summary and Future Research}

BI platforms and data analytics techniques are widespread in businesses, where they assist users to sense and interpret the performance of the enterprise as well as its environment and facilitate decision making. In this context, there exist a gap between the insights resulting from these systems and the action(s) that the enterprise take to respond to the conditions. This paper proposed a methodology to fill this gap and to facilitate enterprise adaptiveness. The methodology synthesizes several existing modeling frameworks to provide a step-by-step coherent way of closing the loop between sense and response in an adaptive enterprise. Applicability of this methodology is illustrated in a hypothetical business setting.

The recent industry trend in "embedded BI" demonstrates increasing recognition of the benefits of adopting a closed-loop sense-and-respond paradigm. However, current solutions are typically limited to a single software application and aim at business process optimization and do not offer strategic enterprise modeling. The current proposal incorporates high level business modeling to support reasoning about alternative actions. The response actions include new sensing mechanisms (BI requirements) in the ongoing evolution of the sense-andrespond loops. The approach is not limited to a single application or business process, but it is intended to be enterprise-wide in scope, to be applied at all levels of business responsibility and performance management.

This research is part of a broader research agenda whose goal is to develop an adaptive enterprise architecture framework. This framework would include modeling, analysis and design tools and techniques to address adaptive enterprise requirements. Future research include implementing and evaluating the proposed methodology in a real case study. Further investigations on the understandability and communicability of the modeling concepts in proposed methodology should be made in future studies. Also, it should be examined how the proposed 
methodology increases the speed of reaction in the enterprise. In this paper, we showed the application of the methodology for closing closing a sense-andrespond loop at one particular level of iterative design cycles. In a complex organization, design and execution occur at many levels of time scales and scopes, as illustrated in [33]. Future works can address this. Moreover, we anticipate that there would be model libraries for various industries that could be reused as best practices for other market players. In future, the proposed methodology could be extended to include industry specific model libraries and hence to leverage the domain knowledge and existing experiences. Besides the BIM framework could be extended to include additional business level concepts, e.g., concepts from the Business Model Canvas [24], and hence to increase its expressiveness for modeling sense-and-respond loops of the enterprise. Finally, we are planning to extend the proposed methodology with a comprehensive catalog of applications of data mining and analytics techniques in business context. We aim to provide model-based support for assisting users to select the proper data mining and analytics techniques based on their business requirements. This catalog along with the modeling support will address the need of business users community for understandable ways of using data mining in an adaptive enterprise context. We leave these extensions to future work.

\section{References}

1. Barone, D., Peyton, L., Rizzolo, F., Amyot, D., Mylopoulos, J.: Towards model-based support for managing organizational transformation. In: Babin, G., Stanoevska-Slabeva, K., Kropf, P. (eds.) MCETECH 2011. LNBIP, vol. 78, pp. 17-31. Springer, Heidelberg (2011)

2. Barone, D., Topaloglou, T., Mylopoulos, J.: Business intelligence modeling in action: A hospital case study. In: Ralyté, J., Franch, X., Brinkkemper, S., Wrycza, S. (eds.) CAiSE 2012. LNCS, vol. 7328, pp. 502-517. Springer, Heidelberg (2012)

3. Barone, D., Yu, E., Won, J., Jiang, L., Mylopoulos, J.: Enterprise modeling for business intelligence. In: van Bommel, P., Hoppenbrouwers, S., Overbeek, S., Proper, E., Barjis, J. (eds.) PoEM 2010. LNBIP, vol. 68, pp. 31-45. Springer, Heidelberg (2010)

4. Buckley, S., Ettl, M., Lin, G., Wang, K.-Y.: Sense and respond business performance management. In: Supply Chain Management on Demand, pp. 287-311. Springer (2005)

5. Chen, H., Chiang, R.H., Storey, V.C.: Business intelligence and analytics: from big data to big impact. MIS Quarterly 36(4), 1165-1188 (2012)

6. Chowdhary, P., Bhaskaran, K., Caswell, N.S., Chang, H., Chao, T., Chen, S.-K., Dikun, M., Lei, H., Jeng, J.-J., Kapoor, S., et al.: Model driven development for business performance management. IBM Systems Journal 45(3), 587-605 (2006)

7. Chung, L., Nixon, B.A., Yu, E., Mylopoulos, J.: Non-functional requirements in software engineering. Kluwer Academic Publishers (2000)

8. Barone, L.J.D., Mylopoulos, J., Amyot, D.: The business intelligence model: Strategic modelling. Technical report, University of Toronto (April 2010)

9. Dardenne, A., Van Lamsweerde, A., Fickas, S.: Goal-directed requirements acquisition. Science of Computer Programming 20(1), 3-50 (1993) 
10. Richard Dealtry, T.: Dynamic SWOT Analysis: Developer's Guide. Intellectual Partnerships (1992)

11. Haeckel, S.H.: Adaptive enterprise: Creating and leading sense-and-respond organizations. Harvard Business Press (1999)

12. Haeckel, S.H.: Peripheral vision: Sensing and acting on weak signals: Making meaning out of apparent noise: The need for a new managerial framework. Long Range Planning 37(2), 181-189 (2004)

13. Haeckel, S.H.: Adaptive enterprise design: the sense-and-respond model. Strategy \& Leadership 23(3), 6-42 (1995)

14. Horkoff, J., Barone, D., Jiang, L., Yu, E., Amyot, D., Borgida, A., Mylopoulos, J.: Strategic business modeling: representation and reasoning. In: Software \& Systems Modeling, pp. 1-27 (2012)

15. Horkoff, J., Borgida, A., Mylopoulos, J., Barone, D., Jiang, L., Yu, E., Amyot, D.: Making Data Meaningful: The Business Intelligence Model and Its Formal Semantics in Description Logics. In: Meersman, R., et al. (eds.) OTM 2012, Part II. LNCS, vol. 7566, pp. 700-717. Springer, Heidelberg (2012)

16. Kaplan, R.S., et al.: Strategy maps: Converting intangible assets into tangible outcomes. Harvard Business Press (2004)

17. Kaplan, R.S., Norton, D.P., Dorf, R.C., Raitanen, M.: The balanced scorecard: translating strategy into action, vol. 4. Harvard Business School Press, Boston (1996)

18. Kapoor, S., Bhattacharya, K., Buckley, S., Chowdhary, P., Ettl, M., Katircioglu, K., Mauch, E., Phillips, L.: A technical framework for sense-and-respond business management. IBM Systems Journal 44(1), 5-24 (2005)

19. Kapoor, S., Binney, B., Buckley, S., Chang, H., Chao, T., Ettl, M., Luddy, E.N., Ravi, R.K., Yang, J.: Sense-and-respond supply chain using model-driven techniques. IBM Systems Journal 46(4), 685-702 (2007)

20. LaValle, S., Hopkins, M., Lesser, E., Shockley, R., Kruschwitz, N.: Analytics: The new path to value. how the smartest organizations are embedding analytics to transform insights into action. MIT Sloan Management Review (2010)

21. Maté, A., Trujillo, J.: Incorporating traceability in conceptual models for data warehouses by using MDA. In: Jeusfeld, M., Delcambre, L., Ling, T.-W. (eds.) ER 2011. LNCS, vol. 6998, pp. 459-466. Springer, Heidelberg (2011)

22. Maté, A., Trujillo, J.: A trace metamodel proposal based on the model driven architecture framework for the traceability of user requirements in data warehouses. In: Information Systems, pp. 753-766 (2012)

23. Nguyen, T.M., Schiefer, J., Tjoa, M.: Sense \& response service architecture (saresa): an approach towards a real-time business intelligence solution and its use for a fraud detection application. In: Proceedings of the 8th ACM International Workshop on Data Warehousing and OLAP, pp. 77-86. ACM (2005)

24. Osterwalder, A., Pigneur, Y.: Business model generation: a handbook for visionaries, game changers, and challengers. Wiley (2010)

25. Panian, Z.: Actionable business intelligence: how to make it available through service-oriented architectures. In: 2nd WSEAS International Conference on Computer Engineering and Applications, CEA 2008 (2008)

26. Panian, Z.: Just-in-time business intelligence and real-time decisioning. In: Proceedings of the 9th WSEAS International Conference on Applied Informatics and Communications, AIC 2009, pp. 106-111 (2009)

27. Rizzolo, F., Kiringa, I., Pottinger, R., Wong, K.: The conceptual integration modeling framework: Abstracting from the multidimensional model. arXiv preprint arXiv:1009.0255 (2010) 
28. $\mathrm{Yu}, \mathrm{E}$.: Modelling strategic relationships for process reengineering. $\mathrm{PhD}$ thesis, Toronto, Ont, Canada (1995)

29. Yu, E.: Towards modelling and reasoning support for early-phase requirements engineering. In: Proceedings of the Third IEEE International Symposium on Requirements Engineering, pp. 226-235. IEEE (1997)

30. Yu, E., Amyot, D., Mussbacher, G., Franch, X., Castro, J.: Practical applications of $i^{*}$ in industry: The state of the art (mini-tutorial). In: 21st IEEE International Requirements Engineering Conference. IEEE CS (to appear, 2013)

31. Yu, E., Deng, S., Sasmal, D.: Enterprise architecture for the adaptive enterprise A vision paper. In: Aier, S., Ekstedt, M., Matthes, F., Proper, E., Sanz, J.L. (eds.) PRET 2012 and TEAR 2012. LNBIP, vol. 131, pp. 146-161. Springer, Heidelberg (2012)

32. Yu, E., Giorgini, P., Maiden, N., Mylopoulos, J.: Social modeling for requirements engineering. MIT Press (2011)

33. Yu, E., Lapouchnian, A., Deng, S.: Adapting to uncertain and evolving enterprise requirements. In: Proc. 7th IEEE International Conference on Research Challenges in Information Science, pp. 155-166 (2013) 